WojCIECH M. ZAJĄCZKOWski (Warszawa)

\title{
EXISTENCE OF SOLUTIONS TO THE (rot, div)-SYSTEM IN $L_{p}$-WEIGHTED SPACES
}

Abstract. The existence of solutions to the elliptic problem $\operatorname{rot} v=w$, $\operatorname{div} v=0$ in a bounded domain $\Omega \subset \mathbb{R}^{3},\left.v \cdot \bar{n}\right|_{S}=0, S=\partial \Omega$ in weighted $L_{p}$-Sobolev spaces is proved. It is assumed that an axis $L$ crosses $\Omega$ and the weight is a negative power function of the distance to the axis. The main part of the proof is devoted to examining solutions of the problem in a neighbourhood of $L$. The existence in $\Omega$ follows from the technique of regularization.

1. Introduction. We consider the elliptic boundary value problem

$$
\begin{array}{ll}
\operatorname{rot} v=w & \text { in } \Omega, \\
\operatorname{div} v=0 & \text { in } \Omega, \\
v \cdot \bar{n}=b & \text { on } S,
\end{array}
$$

where $\Omega \subset \mathbb{R}^{3}$ is a bounded domain, $S=\partial \Omega, S \in C^{2}, \bar{n}$ is the unit outward vector normal to $S$ and the dot denotes the scalar product in $\mathbb{R}^{3}$.

For the solvability of problem (1.1), the following compatibility conditions have to be satisfied:

$$
\begin{aligned}
& \int_{S} b(s) d s=0, \\
& \operatorname{div} w=0 .
\end{aligned}
$$

Let $L$ be an axis passing through $\Omega$.

Our aim is to prove the existence of solutions to problem 1.1 in weighted Sobolev spaces with the weight equal to a power function of the distance to $L$. Therefore, we introduce the weighted Sobolev space $V_{p,-\mu}^{k}(\Omega)$ with the finite

2010 Mathematics Subject Classification: Primary 35J55.

Key words and phrases: elliptic system, estimate in weighted $L_{p}$-Sobolev spaces, existence in weighted $L_{p}$-Sobolev spaces, regularization technique. 
norm

$$
\|u\|_{V_{p,-\mu}^{k}(\Omega)}=\left(\sum_{|\alpha| \leq k} \int_{\Omega}\left|D_{x}^{\alpha} u(x)\right|^{p} \varrho(x)^{p(-\mu-k+|\alpha|)} d x\right)^{1 / p}<\infty,
$$

where $\varrho(x)=\operatorname{dist}\{x, L\}, p \in[1, \infty), \mu \in \mathbb{R}_{+}, k \in \mathbb{N} \cup\{0\}$. Moreover, we introduce the notation

$$
H_{-\mu}^{k}(\Omega)=V_{2,-\mu}^{k}(\Omega), \quad L_{p,-\mu}(\Omega)=V_{p,-\mu}^{0}(\Omega) .
$$

The main result of this paper is the following

THEOREM 1.1. Assume the compatibility conditions 1.2), 1.3). Assume that $w \in V_{p,-\mu}^{k}(\Omega), b \in V_{p,-\mu}^{k+1-1 / p}(S), \mu \in \mathbb{R}_{+}, \mu \notin \mathbb{Z}, k \in \mathbb{N} \cup\{0\}, p \in[2, \infty)$, $S \in C^{2}$. Then there exists a solution to problem 1.1 such that $v \in V_{p,-\mu}^{k+1}(\Omega)$ and

$$
\|v\|_{V_{p,-\mu}^{k+1}(\Omega)} \leq c\left(\|w\|_{V_{p,-\mu}^{k}(\Omega)}+\|b\|_{V_{p,-\mu}^{k+1-1 / p}(S)}\right)
$$

where $c$ does not depend on $v, w, b$.

To prove Theorem 1.1 we need [4] and the following result.

THEOREM 1.2 (see [7]). Assume the compatibility conditions (1.2), (1.3). Assume that $w \in H_{-\mu}^{k}(\Omega), b \in H_{-\mu}^{k+1 / 2}(S), \mu \in \mathbb{R}_{+}, \mu \notin \mathbb{Z}, k \in \mathbb{N} \cup\{0\}$. Then there exists a solution to problem (1.1) such that

$$
\|v\|_{H_{-\mu}^{k+1}(\Omega)} \leq c\left(\|w\|_{H_{-\mu}^{k}(\Omega)}+\|b\|_{H_{-\mu}^{k+1 / 2}(S)}\right)
$$

where $c$ does not depend on $v, w, b$.

The main step in the proofs of Theorems 1.1, 1.2 is to obtain an estimate in weighted spaces in neighbourhoods of points of the axis $L$ because estimates in neighbourhoods at a positive distance to the axis are well known (see [1, 6]). We concentrate on local estimates, and a global estimate follows by applying the idea of regularization (see [7, 3]). Restricting our considerations to neighbourhoods of points of $L$ we showed in [7] that it is sufficient to examine problem (1.1) in neighbourhoods of interior points of $\Omega \cap L$. In this case problem (1.1) can be replaced by the elliptic problem (see [7])

$$
\begin{array}{ll}
-\Delta u=f & \text { in } C_{R, a}, \\
u=0 & \text { on } \partial C_{R, a},
\end{array}
$$

where $C_{R, a}$ is an axially symmetric cylinder with axis of symmetry $L$ located in $\Omega$. Introducing the cylindrical coordinates $r, \varphi, z$ by relations $x_{1}=r \cos \varphi$, $x_{2}=r \sin \varphi, x_{3}=z$, where $x_{1}, x_{2}, x_{3}$ are the Cartesian coordinates such as $L$ is the $x_{3}$-axis we define

$$
C_{R, a}=\left\{x \in \mathbb{R}^{3}: r<R,-a<z<a, \varphi \in[0,2 \pi]\right\}, \quad C_{R, a} \cap S=\emptyset,
$$

and the origin of the Cartesian coordinates is at an interior point of $\Omega \cap L$. 
Now we describe the proofs of Theorems 1.1 and 1.2 underlining their differences. In [7] we examine problem (1.6) by applying the Fourier transforms and using the norms of weighted spaces also in terms of the Fourier transforms. This is connected with the fact that the $L_{2}$-theory is developed in [7]. In this paper we improve the regularity of solutions from [7] by applying the local regularization method (see [5]) and by using the Paley-Littlewood partition of unity so the methods of this paper are totally different from those in [7].

Up to now Theorem 1.1 has not been applied in proofs of existence of regular solutions to the Navier-Stokes equations (see references in [7]). However, in more delicate proofs based on the $L_{p}$-approach Theorem 1.1 will be needed.

2. Notation. Using the cylindrical coordinates we introduce the weighted spaces

$$
V_{p,-\mu}^{k}\left(\mathbb{R}^{3}\right)=\left\{u:\left(\sum_{|\alpha| \leq k} \int_{\mathbb{R}^{3}}\left|D_{x}^{\alpha} u(x)\right|^{p} r^{p(-\mu-k+|\alpha|)} d x\right)^{1 / p}<\infty\right\} .
$$

3. Localization of problem (1.1). We are looking for solutions to problem (1.1) in the form (see [7])

$$
v=\nabla \varphi+u,
$$

where $\varphi$ is a solution to the problem

$$
\Delta \varphi=0,\left.\quad \bar{n} \cdot \nabla \varphi\right|_{S}=b
$$

and $u$ satisfies

$$
\begin{array}{ll}
\operatorname{rot} u=w & \text { in } \Omega, \\
\operatorname{div} u=0 & \text { in } \Omega, \\
\left.u \cdot \bar{n}\right|_{S}=0 . &
\end{array}
$$

From [2] we know that $(3.3)_{2,3}$ imply the existence of a vector field $e$ such that

$$
u=\operatorname{rot} e, \quad \operatorname{div} e=0,\left.\quad e \cdot \bar{\tau}\right|_{S}=0,
$$

where $\bar{\tau}$ is a tangent vector to $S$.

In view of (3.4) problem (3.3) takes the form

$$
-\Delta e=w,\left.\quad e \cdot \bar{\tau}\right|_{S}=0,\left.\quad \operatorname{div} e\right|_{S}=0 .
$$

Localizing problems $(3.2)$ and 3.5 by a smooth function from a partition of unity we obtain problem (1.6). The lower order terms which appear in this procedure will be estimated by applying the regularization method. 
4. Regularity near the axis $L$ in the $L_{p}$-approach. After localization of problems (3.2) and (3.5) to a neighbourhood of an internal point of $L$ or a point where $L$ meets $S$ we can replace them by the problem

$$
\begin{array}{ll}
-\Delta u=f & \text { in } \Omega, \\
u=0 & \text { on } \partial \Omega,
\end{array}
$$

where $\Omega=C_{R, a}$.

Lemma 4.1 (local regularity; see [5]). Let $B_{r}=\left\{x_{3} \in \mathbb{R}:\left|x_{3}\right|<r\right\}$, $\xi_{i}=\xi_{i}\left(x^{\prime}\right), x^{\prime}=\left(x_{1}, x_{2}\right), \xi_{i} \in C_{0}^{\infty}\left(\mathbb{R}^{2}\right), i=1,2, \xi_{1} \xi_{2}=\xi_{1}, \operatorname{supp} \xi_{2} \subset\left\{x^{\prime}:\right.$ $\left.c_{1}<\left|x^{\prime}\right|<c_{2}\right\}$. Then for every $u \in W_{p}^{l+2}\left(\mathbb{R}^{2} \times B_{2}\right)$ the following inequality holds:

$$
\left\|\xi_{1} u\right\|_{W_{p}^{l+2}\left(\mathbb{R}^{2} \times B_{1}\right)} \leq c\left(\left\|\xi_{2} \Delta u\right\|_{W_{p}^{l}\left(\mathbb{R}^{2} \times B_{2}\right)}+\left\|\xi_{2} u\right\|_{L_{p_{1}}\left(\mathbb{R}^{2} \times B_{2}\right)}\right),
$$

for any $p_{1} \in[1, \infty]$, where $c$ does not depend on $u$.

Let us introduce partitions of unity $\left\{\zeta_{j}\right\}_{j=-\infty}^{\infty},\left\{\sigma_{j}\right\}_{j=-\infty}^{\infty}$ such that

$$
\begin{aligned}
& \operatorname{supp} \zeta_{j} \subset\left\{x^{\prime}: 2^{j-1}<\left|x^{\prime}\right|<2^{j+1}\right\}, \\
& \operatorname{supp} \sigma_{j} \subset\left\{x^{\prime}: 2^{j-2}<\left|x^{\prime}\right|<2^{j+2}\right\},
\end{aligned}
$$

$\sigma_{j}, \zeta_{j} \in C^{\infty}\left(\mathbb{R}^{2}\right), \zeta_{j} \sigma_{j}=\zeta_{j}$, and for all multiindices $\alpha$,

$$
\left|D^{\alpha} \zeta_{j}\right|+\left|D^{\alpha} \sigma_{j}\right| \leq c_{\alpha} 2^{-j|\alpha|} .
$$

LEMma 4.2. Let $\beta \in \mathbb{R}$. Then for any $u \in W_{p}^{l+2}\left(\left\{2^{j-2}<\left|x^{\prime}\right|<2^{j+2}\right\} \times \mathbb{R}^{1}\right)$ the following inequality holds,

$$
\left\|\zeta_{j} u\right\|_{V_{p, \beta}^{l+2}\left(\mathbb{R}^{2} \times \mathbb{R}^{1}\right)} \leq c\left(\left\|\sigma_{j} \Delta u\right\|_{V_{p, \beta}^{l}\left(\mathbb{R}^{2} \times \mathbb{R}^{1}\right)}+\left\|\sigma_{j} u\right\|_{L_{p, \beta-l-2}\left(\mathbb{R}^{2} \times \mathbb{R}^{1}\right)}\right),
$$

where $c$ does not depend on $u$ and $j$.

Proof. Let $K=\left\{x^{\prime}: 1<\left|x^{\prime}\right|<2\right\}, B=\left\{x_{3}:\left|x_{3}\right|<2\right\}, K_{\mu}=\left\{x^{\prime}: 2^{\mu}<\right.$ $\left.\left|x^{\prime}\right|<2^{\mu+1}\right\}, B_{\mu}=\left\{x_{3}:\left|x_{3}\right|<2^{\mu+1}\right\}$. From 4.2 we have

$$
\begin{aligned}
\sum_{|\alpha|=0}^{l+2}\left\|D^{\alpha}\left(\zeta_{j} u\right)\right\|_{L_{p}(K \times B)} \leq & c \sum_{|\alpha|=0}^{l}\left\|D^{\alpha}\left(\sigma_{j} \Delta u\right)\right\|_{L_{p}(2 K \times 2 B)} \\
& +c\left\|\sigma_{j} u\right\|_{L_{p}(2 K \times 2 B)},
\end{aligned}
$$

where $2 K=\left\{x^{\prime}: 1 / 2<\left|x^{\prime}\right|<4\right\}, 2 B=\left\{x_{3}:\left|x_{3}\right|<4\right\}$. In view of scaling $x \mapsto 2^{\mu} x$ we obtain

$$
\begin{aligned}
& \sum_{|\alpha|=0}^{l+2} 2^{\mu(|\alpha|-2)}\left\|D^{\alpha}\left(\zeta_{j} u\right)\right\|_{L_{p}\left(K_{\mu} \times B_{\mu}\right)} \\
& \leq c \sum_{|\alpha|=0}^{l} 2^{\mu|\alpha|}\left\|D^{\alpha} \sigma_{j} \Delta u\right\|_{L_{p}\left(2 K_{\mu} \times 2 B_{\mu}\right)}+c 2^{-2 \mu}\left\|\sigma_{j} u\right\|_{L_{p}\left(2 K_{\mu} \times 2 B_{\mu}\right)} .
\end{aligned}
$$


Multiplying 4.5 by $2^{(\beta-l) \mu}$, taking the $p$ th power, summing over $\mu$ and using that $r \sim 2^{\mu}$ on the set $K_{\mu}$, we obtain

$$
\begin{aligned}
& \sum_{|\alpha|=0}^{l+2}\left\|r^{\beta-l-2+|\alpha|} D^{\alpha}\left(\zeta_{j} u\right)\right\|_{L_{p}\left(\mathbb{R}^{3}\right)} \\
& \quad \leq c \sum_{|\alpha|=0}^{l}\left\|r^{\beta-l+|\alpha|} D^{\alpha}\left(\sigma_{j} \Delta u\right)\right\|_{L_{p}\left(\mathbb{R}^{3}\right)}+c\left\|r^{\beta-l-2} \sigma_{j} u\right\|_{L_{p}\left(\mathbb{R}^{3}\right)} .
\end{aligned}
$$

This implies (4.3) and concludes the proof.

Summing up 4.3 with respect to $j$ yields

$$
\|u\|_{V_{p, \beta}^{l+2}\left(\mathbb{R}^{3}\right)} \leq c\|\Delta u\|_{V_{p, \beta}^{l}\left(\mathbb{R}^{3}\right)}+c\|u\|_{V_{p, \beta-l-2}^{0}\left(\mathbb{R}^{3}\right)} .
$$

Let

$$
P\left(\partial_{x^{\prime}}, \partial_{x_{3}}\right)=-\Delta, \quad P\left(\partial_{x^{\prime}}, \eta\right)=-\Delta^{\prime}+\eta^{2},
$$

where $\Delta^{\prime}=\partial_{x_{1}}^{2}+\partial_{x_{2}}^{2}$. Let us denote by $A(\eta): V_{p, \beta}^{l+2}\left(\mathbb{R}^{2}\right) \rightarrow V_{p, \beta}^{l}\left(\mathbb{R}^{2}\right)$ the operator of the problem

$$
\begin{array}{ll}
P\left(\partial_{x^{\prime}}, \eta\right) u=f & \text { in } \mathbb{R}^{2}, \\
u=0 & \text { on } S_{R}^{1},
\end{array}
$$

where $S_{R}^{1}=\left\{x^{\prime}:\left|x^{\prime}\right|=R\right\}$.

First, we consider the problem

$$
\begin{array}{ll}
P\left(\partial_{x^{\prime}}, 0\right) u=f & \text { in } \mathbb{R}^{2}, \\
u=0 & \text { on } S_{R}^{1} .
\end{array}
$$

Passing to the polar coordinates $(r, \varphi)$, next to the variable $\tau=-\ln r$ and applying the Fourier transform with respect to $\tau$ we have

$$
\begin{aligned}
& \bar{P}\left(\partial_{\varphi}, \lambda\right) \tilde{u}=\widetilde{e^{2 \tau} f}, \\
& \left.u\right|_{\varphi=0}=\left.u\right|_{\varphi=2 \pi}, \\
& \left.u_{, \varphi}\right|_{\varphi=0}=\left.u_{, \varphi}\right|_{\varphi=2 \pi},
\end{aligned}
$$

and

$$
\tilde{u}(\lambda, \varphi)=\int_{\mathbb{R}^{1}} u(\tau, \varphi) e^{-i \lambda \tau} d \tau .
$$

On the line $\operatorname{im} \lambda=\beta-1 \notin \mathbb{Z}$ there is no eigenvalue of problem 4.10). Moreover, $\operatorname{ker} A(\eta)$ and coker $A(\eta)$ are trivial in the spaces $V_{2, \beta}^{0}\left(\mathbb{R}^{2}\right), \beta \notin \mathbb{Z}$. Thus the operator $A(\eta)^{-1}$ is defined in $V_{2, \beta}^{0}\left(\mathbb{R}^{2}\right), \beta \notin \mathbb{Z}$. 
Lemma 4.3. For any $\eta \in \mathbb{R}^{1} \backslash\{0\}$ and any $f \in V_{2, \beta}^{0}\left(\mathbb{R}^{2}\right), \beta \notin \mathbb{Z}$, the following inequality holds:

$$
\sum_{\nu=0}^{2}|\eta|^{\nu}\left\|\partial_{\eta}^{\gamma} A(\eta)^{-1} f\right\|_{V_{2, \beta}^{2-\nu}\left(\mathbb{R}^{2}\right)} \leq c|\eta|^{-\gamma}\|f\|_{V_{2, \beta}^{0}\left(\mathbb{R}^{2}\right)} .
$$

Proof. We have

$$
\partial_{\eta}^{\gamma} A(\eta)^{-1}=\sum_{1 \leq q \leq \gamma} \sum_{\alpha_{1}+\cdots+\alpha_{q}=\gamma} c_{\alpha_{1} \ldots \alpha_{q}} A^{-1} \partial_{\eta}^{\alpha_{1}} P A^{-1} \partial_{\eta}^{\alpha_{2}} P \ldots A^{-1} \partial_{\eta}^{\alpha_{q}} P A^{-1},
$$

where $c_{\alpha_{1} \ldots \alpha_{q}}$ are some constant coefficients. Since

$$
\sum_{\nu=0}^{2}|\eta|^{\nu}\|u\|_{V_{2, \beta}^{2-\nu}\left(\mathbb{R}^{2}\right)} \leq c\left\|P\left(\partial_{x^{\prime}}, \eta\right) u\right\|_{V_{2, \beta}^{0}\left(\mathbb{R}^{2}\right)},
$$

and

$$
\left\|\partial_{\eta}^{\gamma} P\left(\partial_{x^{\prime}}, \eta\right) A(\eta)^{-1} f\right\|_{V_{2, \beta}^{0}\left(\mathbb{R}^{2}\right)} \leq c \sum_{\nu=0}^{2-\gamma}|\eta|^{\nu}\left\|A(\eta)^{-1} f\right\|_{V_{2, \beta}^{2-\gamma-\nu}\left(\mathbb{R}^{2}\right)},
$$

we obtain

$$
\left\|\partial_{\eta}^{\gamma} P\left(\partial_{x^{\prime}}, \eta\right) A(\eta)^{-1} f\right\|_{V_{2, \beta}^{0}\left(\mathbb{R}^{2}\right)} \leq c|\eta|^{-\gamma}\|f\|_{V_{2, \beta}^{0}\left(\mathbb{R}^{2}\right)} .
$$

Therefore (4.11) is proved. This concludes the proof.

LEMMA 4.4. Let the assumptions of Lemma 4.3 be satisfied. Let $\sigma_{\mu}, \zeta_{\nu}$ be the functions introduced after Lemma 4.1. Then for all $\eta \neq 0$ the estimate

$$
\left\|\sigma_{\mu} A(\eta)^{-1} \zeta_{\nu}\right\|_{V_{2, \beta}^{0}\left(\mathbb{R}^{2}\right) \rightarrow V_{2, \beta}^{0}\left(\mathbb{R}^{2}\right)} \leq c 2^{-\varepsilon|\mu-\nu|+2 \mu}
$$

holds for $\beta \notin \mathbb{Z}$ and $\varepsilon>0$ sufficiently small.

Proof. Observe that the operator $\sigma_{\mu} A(\eta)^{-1} \zeta_{\nu}$ maps functions with support in $\operatorname{supp} \zeta_{\nu}$ to functions with support in $\operatorname{supp} \sigma_{\mu}$.

First we examine the operator $r^{ \pm \varepsilon} A(\eta) r^{\mp \varepsilon}$. Since $A(\eta)=-\Delta^{\prime}+\eta^{2}$ and $A(\eta)$ maps $V_{2, \beta}^{2}\left(\mathbb{R}^{2}\right)$ into $V_{2, \beta}^{0}\left(\mathbb{R}^{2}\right)$ we have for small $\varepsilon$,

$$
\begin{aligned}
& \left\|r^{ \pm \varepsilon} A(\eta) r^{\mp \varepsilon} u\right\|_{V_{2, \beta}^{0}\left(\mathbb{R}^{2}\right)}=\left\|r^{ \pm \varepsilon}\left(-\Delta^{\prime}+\eta^{2}\right) r^{\mp \varepsilon} u\right\|_{V_{2, \beta}^{0}\left(\mathbb{R}^{2}\right)} \\
& \leq\left\|\left(-\Delta^{\prime}+\eta^{2}\right) u\right\|_{V_{2, \beta}^{0}\left(\mathbb{R}^{2}\right)}+\varepsilon\left\|r^{\mp \varepsilon} r^{ \pm \varepsilon-1} \nabla^{\prime} r \nabla^{\prime} u\right\|_{V_{2, \beta}^{0}\left(\mathbb{R}^{2}\right)} \\
& \quad+\varepsilon(1-\varepsilon)\left\|r^{\mp \varepsilon} r^{ \pm \varepsilon-2}\left|\nabla^{\prime} r\right|^{2} u\right\|_{V_{2, \beta}^{0}\left(\mathbb{R}^{2}\right)}+\varepsilon\left\|r^{\mp \varepsilon} r^{ \pm \varepsilon-1} \Delta^{\prime} r u\right\|_{V_{2, \beta}^{0}\left(\mathbb{R}^{2}\right)} \\
& \leq\|A(\eta) u\|_{V_{2, \beta}^{0}\left(\mathbb{R}^{2}\right)}+c \varepsilon\|u\|_{V_{2, \beta-1}^{1}\left(\mathbb{R}^{2}\right)} .
\end{aligned}
$$


Conversely,

$$
\begin{aligned}
\|A(\eta) u\|_{V_{2, \beta}^{0}\left(\mathbb{R}^{2}\right)} & =\left\|r^{\mp \varepsilon} r^{ \pm \varepsilon} A(\eta) u\right\|_{V_{2, \beta}^{0}\left(\mathbb{R}^{2}\right)} \\
& =\left\|r^{\mp \varepsilon} r^{ \pm \varepsilon}\left(-\Delta^{\prime}+\eta^{2}\right) u\right\|_{V_{2, \beta}^{0}\left(\mathbb{R}^{2}\right)} \\
& \leq\left\|r^{\mp \varepsilon}\left(-\Delta^{\prime}+\eta^{2}\right) r^{ \pm \varepsilon} u\right\|_{V_{2, \beta}^{0}\left(\mathbb{R}^{2}\right)}+c \varepsilon\|u\|_{V_{2, \beta-1}^{1}\left(\mathbb{R}^{2}\right)} .
\end{aligned}
$$

Hence for small $\varepsilon$, the norms of the operators $r^{ \pm \varepsilon} A(\eta) r^{\mp \varepsilon}$ and $A(\eta)$ are close to each other because

$$
\left|\sup _{\|u\|_{V_{2, \beta}^{2}\left(\mathbb{R}^{2}\right)} \leq 1}\left\|r^{ \pm \varepsilon} A(\eta) r^{\mp \varepsilon} u\right\|_{V_{2, \beta}^{0}\left(\mathbb{R}^{2}\right)}-\sup _{\|u\|_{V_{2, \beta}^{2}\left(\mathbb{R}^{2}\right)} \leq 1}\|A(\eta) u\|_{V_{2, \beta}^{0}\left(\mathbb{R}^{2}\right)}\right| \leq c \varepsilon .
$$

Therefore for $\beta \pm \varepsilon \notin \mathbb{Z}$ and $\varepsilon$ sufficiently small the operator $A(\eta)$ is an isomorphism from $V_{2, \beta \pm \varepsilon}^{2}\left(\mathbb{R}^{2}\right)$ into $V_{2, \beta \pm \varepsilon}^{0}\left(\mathbb{R}^{2}\right)$. Let $u_{\mu}=u \sigma_{\mu}$ and $f_{\nu}=f \zeta_{\nu}$. Then we examine the problem

$$
P\left(\partial_{x^{\prime}}, \eta\right) u=f_{\nu}
$$

Since the operator $A(\eta)^{-1}$ is defined we have

$$
u_{\mu}=\sigma_{\mu} A^{-1}(\eta) f_{\nu}
$$

In view of 4.11 we obtain

$$
\begin{aligned}
\left\|u_{\mu}\right\|_{V_{2, \beta}^{2}\left(\mathbb{R}^{2}\right)} & \leq c 2^{\varepsilon \mu}\left\|u_{\mu}\right\|_{V_{2, \beta-\varepsilon}^{2}\left(\mathbb{R}^{2}\right)} \\
& \leq c 2^{\varepsilon \mu}\left\|f_{\nu}\right\|_{V_{2, \beta-\varepsilon}^{0}\left(\mathbb{R}^{2}\right)} \leq c 2^{\varepsilon(\mu-\nu)}\left\|f_{\nu}\right\|_{V_{2, \beta}^{0}\left(\mathbb{R}^{2}\right)} .
\end{aligned}
$$

Since

$$
\left\|u_{\mu}\right\|_{V_{2, \beta}^{2}\left(\mathbb{R}^{2}\right)} \geq c 2^{-2 \mu}\left\|u_{\mu}\right\|_{V_{2, \beta}^{0}\left(\mathbb{R}^{2}\right)}
$$

we obtain

$$
\left\|u_{\mu}\right\|_{V_{2, \mu}^{0}\left(\mathbb{R}^{2}\right)} \leq c 2^{\varepsilon(\mu-\nu)+2 \mu}\left\|f_{\nu}\right\|_{V_{2, \beta}^{0}\left(\mathbb{R}^{2}\right)},
$$

where for $\mu>\nu$ we replace $\varepsilon$ by $-\varepsilon, \varepsilon>0$ and for $\mu<\nu$ we take $\varepsilon$. Then we have

$$
\left\|u_{\mu}\right\|_{V_{2, \beta}^{0}\left(\mathbb{R}^{2}\right)} \leq c 2^{-\varepsilon|\mu-\nu|+2 \mu}\left\|f_{\nu}\right\|_{V_{2, \beta}^{0}\left(\mathbb{R}^{2}\right)} .
$$

From 4.15 we derive 4.12. This concludes the proof.

LEMmA 4.5. Let the assumptions of Lemma 4.4 be satisfied. Then the operator $P$ of the problem

$$
\begin{aligned}
& P\left(\partial_{x^{\prime}}, \partial_{x_{3}}\right) u=f \\
& \left.u\right|_{S_{R}}=0
\end{aligned}
$$

is an isomorphism

$$
P: V_{2, \beta}^{2}\left(\mathbb{R}^{3}\right) \rightarrow V_{2, \beta}^{0}\left(\mathbb{R}^{3}\right)
$$


The lemma follows from the estimate

$$
\|u\|_{V_{2, \beta}^{2}\left(\mathbb{R}^{3}\right)} \leq c\|f\|_{V_{2, \beta}^{0}\left(\mathbb{R}^{3}\right)}, \quad \beta \notin \mathbb{Z},
$$

and the fact that ker $P$ in $V_{2, \beta}^{2}\left(\mathbb{R}^{3}\right)$ and coker $P$ in $V_{2, \beta}^{0}\left(\mathbb{R}^{3}\right)$ are trivial.

To prove the next lemma we need the following Marcinkiewicz-Mikhlin type result (see [2, Ch. 11, §11]):

Lemma 4.6. Let $L_{p}\left(\mathbb{R}^{d} ; H\right)$ be the space of functions with the finite norm

$$
\|f\|_{L_{p}\left(\mathbb{R}^{d} ; H\right)}=\left(\int_{\mathbb{R}^{d}}\|f(z)\|_{H}^{p} d z\right)^{1 / p}<\infty,
$$

where $H$ is a Hilbert space. Let $M(\xi), \xi \in \mathbb{R}^{d}$, be a bounded function in $\mathbb{R}^{d}$ acting as a multiplier. Let for $s=0, \ldots, d, i_{k} \neq i_{l}$,

$$
|\xi|^{s}\left\|\frac{\partial^{s} M}{\partial \xi_{i_{1}} \ldots \partial \xi_{i_{s}}}(\xi)\right\|_{H \rightarrow H} \leq \text { const. }
$$

Then $F_{\xi \rightarrow z}^{-1} M(\xi) F_{z \rightarrow \xi}$, where $F$ is the Fourier transform in $\mathbb{R}^{d}$, is a continuous operator in $L_{p}\left(\mathbb{R}^{d} ; H\right)$.

LEMma 4.7. Let the assumptions of Lemma 4.3 be satisfied. Let $u_{\nu} \in$ $V_{2, \beta}^{2}\left(\mathbb{R}^{3}\right)$ be a solution to the problem

$$
P\left(\partial_{x^{\prime}}, \partial_{x_{3}}\right) u_{\nu}=\zeta_{\nu} f .
$$

Then

$$
\begin{aligned}
\int_{\mathbb{R}^{1}}\left(\int_{\mathbb{R}^{2}} r^{2 \beta}\left|\sigma_{\mu}\left(x^{\prime}\right) u_{\nu}\left(x^{\prime}, x_{3}\right)\right|^{2} d x^{\prime}\right)^{p / 2} d x_{3} \\
\quad \leq c 2^{-p \varepsilon|\mu-\nu|+2 \mu p} \int_{\mathbb{R}^{1}}\left(\int_{\mathbb{R}^{2}} r^{2 \beta}\left|\zeta_{\nu}\left(x^{\prime}\right) f\left(x^{\prime}, x_{3}\right)\right|^{2} d x^{\prime}\right)^{p / 2} d x_{3},
\end{aligned}
$$

where $c$ does not depend on $u$ and $f$.

Proof. We have

$$
u_{\nu}=F_{\eta \rightarrow x_{3}}^{-1} A(\eta)^{-1} F_{x_{3} \rightarrow \eta} \zeta_{\nu} f,
$$

where $F$ is the Fourier transform in $\mathbb{R}^{1}$.

By Lemma $4.6 F_{\eta \rightarrow x_{3}}^{-1} M(\eta) F_{x_{3} \rightarrow \eta}$, where $M(\eta)=\sigma_{\mu} A(\eta)^{-1} \zeta_{\nu}$, is a continuous operator in $L_{p}\left(\mathbb{R}^{1} ; V_{2, \beta}^{0}\left(\mathbb{R}^{2}\right)\right)$. Then by 4.12 we obtain 4.18). This concludes the proof.

Lemma 4.8. Let the assumptions of Lemma 4.3 be satisfied. Let $u_{\nu}$ be defined in the assumptions of Lemma 4.7. Then for $p \geq 2$ we have 


$$
\begin{aligned}
& \int_{\mathbb{R}^{3}} r^{p(\beta-1)-2}\left|\zeta_{\mu}\left(x^{\prime}\right) u_{\nu}\left(x^{\prime}, x_{3}\right)\right|^{p} d x^{\prime} d x_{3} \\
& \leq c 2^{-\varepsilon|\mu-\nu| p} \int_{\mathbb{R}^{3}} r^{p(\beta+1)-2}\left|\zeta_{\nu}\left(x^{\prime}\right) f\left(x^{\prime}, x_{3}\right)\right|^{p} d x^{\prime} d x_{3},
\end{aligned}
$$

where $c$ does not depend on $u$ and $f$.

Proof. By the Hölder inequality the r.h.s. of 4.18 is estimated by

$$
\begin{array}{rl}
c 2^{-p \varepsilon|\mu-\nu|+2 \mu p} \int_{\mathbb{R}^{1}} & d x_{3}\left[\left|\operatorname{supp} \zeta_{\nu}\right|^{(p-2) / p}\left(\int_{\mathbb{R}^{2}} r^{p \beta}\left|\zeta_{\nu} f\right|^{p} d x^{\prime}\right)^{2 / p}\right]^{p / 2} \\
& \leq c 2^{-p \varepsilon|\mu-\nu|+2 \mu p} \int_{\mathbb{R}^{1}} d x_{3} 2^{\nu(p-2)} \int_{\mathbb{R}^{2}} r^{p \beta}\left|\zeta_{\nu} f\right|^{p} d x^{\prime} \\
& \leq c 2^{-p \varepsilon|\mu-\nu|+2 \mu p} \int_{\mathbb{R}^{1}} d x_{3} \int_{\mathbb{R}^{2}} r^{p(\beta+1)-2}\left|\zeta_{\nu} f\right|^{p} d x^{\prime},
\end{array}
$$

where we used that on $\operatorname{supp} \zeta_{\nu}$ we have $r \in\left(2^{\nu-1}, 2^{\nu+1}\right)$.

Divide $\mathbb{R}^{1}$ into segments $Q_{j}$ of length $2^{\mu}$ each. By $2 Q_{j}$ we denote a segment with length $2^{\mu+1}$ which contains $Q_{j}$.

By the Hölder inequality we have

$$
\begin{aligned}
\int_{2 Q_{j} \mathbb{R}^{2}} \int_{\mu}\left|\sigma_{\mu} u_{\nu}\right| d x^{\prime} d x_{3} & \leq \int_{2 Q_{j}}\left|\operatorname{supp} \sigma_{\mu}\right|^{1 / 2}\left(\int_{\mathbb{R}^{2}}\left|\sigma_{\mu} u_{\nu}\right|^{2} d x^{\prime}\right)^{1 / 2} d x_{3} \\
& \leq c \int_{2 Q_{j}} 2^{\mu} d x_{3}\left(\int_{\mathbb{R}^{2}}\left|\sigma_{\mu} u_{\nu}\right|^{2} d x^{\prime}\right)^{1 / 2} \equiv I_{1},
\end{aligned}
$$

where we used that

$$
\operatorname{supp} \sigma_{\mu} \subset\left\{x^{\prime} \in \mathbb{R}^{2}: 2^{\mu-2}<\left|x^{\prime}\right|<2^{\mu+2}\right\} .
$$

Continuing,

$$
I_{1} \leq c \int_{2 Q_{j}} 2^{\mu-\beta \mu}\left(\int_{\mathbb{R}^{2}} r^{2 \beta}\left|\sigma_{\mu} u_{\nu}\right|^{2} d x^{\prime}\right)^{1 / 2} d x_{3} \equiv I_{2},
$$

where we used (4.21) again. Continuing,

$$
\begin{aligned}
& \left(\int_{2 Q_{j}} \int_{\mathbb{R}^{2}}\left|\sigma_{\mu} u_{\nu}\right| d x^{\prime} d x_{3}\right)^{p} \leq 2^{(1-\beta) \mu p}\left[\int_{2 Q_{j}} d x_{3}\left(\int_{\mathbb{R}^{2}} r^{2 \beta}\left|\sigma_{\mu} u_{\nu}\right|^{2} d x^{\prime}\right)^{1 / 2}\right]^{p} \\
& \leq c 2^{(1-\beta) \mu p}\left(\int_{2 Q_{j}} 1^{p^{\prime}} d x_{3}\right)^{p / p^{\prime}}\left(\int_{2 Q_{j}} d x_{3}\left(\int_{\mathbb{R}^{2}} r^{2 \beta}\left|\sigma_{\mu} u_{\nu}\right|^{2} d x^{\prime}\right)^{p / 2}\right) \equiv I_{3},
\end{aligned}
$$

where $1 / p+1 / p^{\prime}=1, p / p^{\prime}=p-1$. Hence, we have

$$
I_{3}=c 2^{(1-\beta) \mu p+\mu(p-1)} \int_{2 Q_{j}} d x_{3}\left(\int_{\mathbb{R}^{3}} r^{2 \beta}\left|\sigma_{\mu} u_{\nu}\right|^{2} d x^{\prime}\right)^{p / 2} .
$$


Therefore, we get

$$
\left(\int_{2 Q_{j}} \int_{\mathbb{R}^{2}}\left|\sigma_{\mu} u_{\nu}\right| d x^{\prime} d x_{3}\right)^{p} \leq c 2^{-\mu p(\beta-2)-\mu} \int_{2 Q_{j}} d x_{3}\left(\int_{\mathbb{R}^{2}} r^{2 \beta}\left|\sigma_{\mu} u_{\nu}\right|^{2} d x^{\prime}\right)^{p / 2} .
$$

From Lemma 4.1 for $|\mu-\nu|>3$ we obtain the inequality

$$
\int_{Q_{j} \mathbb{R}^{2}}\left|\zeta_{\mu} u_{\nu}\right|^{p} d x^{\prime} d x_{3} \leq c 2^{3 \mu(1-p)}\left(\int_{2 Q_{j} \mathbb{R}^{2}}\left|\sigma_{\mu} u_{\nu}\right| d x^{\prime} d x_{3}\right)^{p}
$$

by a scaling argument.

From 4.22 and 4.23 and for $|\mu-\nu|>3$ we obtain

$$
\int_{\mathbb{R}^{3}}\left|\zeta_{\mu} u_{\nu}\right|^{p} d x^{\prime} d x_{3} \leq c 2^{2 \mu-\mu p(\beta-1)} \int_{\mathbb{R}}\left(\int_{\mathbb{R}^{2}} r^{2 \beta}\left|\sigma_{\mu} u_{\nu}\right|^{2} d x^{\prime}\right)^{p / 2} d x_{3} .
$$

In the case $|\mu-\nu| \leq 3$ we have to add on the r.h.s. of (4.22) the expression

$$
c 2^{2 \mu p} \int_{2 Q_{j}} \int_{\mathbb{R}^{2}}\left|\zeta_{\nu} f\right|^{p} d x^{\prime} d x_{3}
$$

Hence, in this case, we have to add the same term on the r.h.s. of (4.24). Using (4.18) we obtain 4.19). This concludes the proof.

To prove the next lemma we need a result on operators in Banach spaces. Let $\mathcal{E}_{0}\left(\mathbb{R}^{3}\right), \mathcal{E}_{1}\left(\mathbb{R}^{3}\right)$ be Banach spaces of functions defined on $\mathbb{R}^{3}$, closed under pointwise multiplication with functions from $C_{0}^{\infty}\left(\mathbb{R}^{2} \backslash\{0\}\right)$. Let $\left\{\zeta_{j}\right\}_{j=-\infty}^{\infty}$ be the partition of unity described above.

Assume that there exist $p$ and $q, 1 \leq p \leq q \leq \infty$, such that for all $u \in \mathcal{E}_{0}$, $v \in \mathcal{E}_{1}$ the following inequalities hold:

$$
\begin{aligned}
& \|u\|_{\mathcal{E}_{0}} \leq c\left(\sum_{j=-\infty}^{\infty}\left\|\zeta_{j} u\right\|_{\mathcal{E}_{0}}^{q}\right)^{1 / q}, \\
& \|v\|_{\mathcal{E}_{1}} \geq c\left(\sum_{j=-\infty}^{\infty}\left\|\zeta_{j} v\right\|_{\mathcal{E}_{1}}^{p}\right)^{1 / p},
\end{aligned}
$$

where $\|\cdot\|_{\mathcal{E}_{i}}$ is the norm of $\mathcal{E}_{i}, i=0,1$.

Lemma 4.9. Let $\theta: \mathcal{E}_{1} \rightarrow \mathcal{E}_{0}$ be a linear operator defined on functions with compact supports such that for some $\varepsilon>0$ and all $\mu, \nu \in \mathbb{Z}$,

$$
\left\|\zeta_{\mu} \theta \zeta_{\nu} v\right\|_{\mathcal{E}_{0}} \leq c e^{-\varepsilon|\mu-\nu|}\left\|\zeta_{\nu} v\right\|_{\mathcal{E}_{1}}
$$

for all $v \in \mathcal{E}_{1}\left(\mathbb{R}^{3}\right)$. Then for any $v \in \mathcal{E}_{1}\left(\mathbb{R}^{3}\right)$ with compact support,

$$
\|\theta v\|_{\mathcal{E}_{0}\left(\mathbb{R}^{3}\right)} \leq c\|v\|_{\mathcal{E}_{1}\left(\mathbb{R}^{3}\right)},
$$

where $c$ does not depend on $v$. 
Proof. From 4.25,

$$
\begin{aligned}
\|\theta v\|_{\mathcal{E}_{0}\left(\mathbb{R}^{3}\right)} & =\left\|\theta\left(\sum_{\nu=-\infty}^{\infty} \zeta_{\nu} v\right)\right\|_{\mathcal{E}_{0}\left(\mathbb{R}^{3}\right)} \leq c\left(\sum_{\mu=-\infty}^{\infty}\left\|\sum_{\nu=-\infty}^{\infty} \xi_{\mu} \theta \xi_{\nu} v\right\|_{\mathcal{E}_{0}\left(\mathbb{R}^{3}\right)}^{q}\right)^{1 / q} \\
& \leq c\left[\sum_{\mu=-\infty}^{\infty}\left(\sum_{\nu=-\infty}^{\infty}\left\|\xi_{\mu} \theta \xi_{\nu} v\right\|_{\mathcal{E}_{0}\left(\mathbb{R}^{3}\right)}\right)^{q}\right]^{1 / q} \cdot
\end{aligned}
$$

Applying 4.27) yields

$$
\left.\|\theta v\|_{\mathcal{E}_{0}\left(\mathbb{R}^{3}\right)} \leq c \sum_{\mu=-\infty}^{\infty}\left(\sum_{\nu=-\infty}^{\infty} e^{-\varepsilon|\mu-\nu|}\left\|\zeta_{\nu} v\right\|_{\mathcal{E}_{1}\left(\mathbb{R}^{3}\right)}\right)^{q}\right]^{1 / q} .
$$

Since the operator of discrete convolution with the kernel $\left\{e^{-|j| \varepsilon}\right\}_{j=-\infty}^{\infty}$ is continuous from $l_{p}$ to $l_{q}$ for $q \geq p$, we have

$$
\|\theta v\|_{\mathcal{E}_{0}\left(\mathbb{R}^{3}\right)} \leq c\left(\sum_{\nu=-\infty}^{\infty}\left\|\zeta_{\nu} v\right\|_{\mathcal{E}_{1}\left(\mathbb{R}^{3}\right)}^{p}\right)^{1 / p} .
$$

Using now 4.26 we obtain 4.28. This concludes the proof.

LEMMA 4.10. Let the assumptions of Lemma 4.4 be satisfied. Let $u \in$ $V_{2, \beta}^{2}\left(\mathbb{R}^{3}\right)$ be a solution of problem 4.16). Then for $p \geq 2$ the inequality

$$
\int_{\mathbb{R}^{3}} r^{p(\beta-1)-2}\left|u\left(x^{\prime}, x_{3}\right)\right|^{p} d x^{\prime} d x_{3} \leq c \int_{\mathbb{R}^{3}} r^{p(\beta+1)-2}\left|f\left(x^{\prime}, x_{3}\right)\right|^{p} d x^{\prime} d x_{3}
$$

is valid, where $c$ does not depend on $u$ and $f$.

Proof. We apply Lemma 4.9 to the inequality of Lemma 4.8 with $q=p$, $\mathcal{E}_{0}=V_{p, \beta-1-2 / p}^{0}\left(\mathbb{R}^{3}\right), \mathcal{E}_{1}=V_{p, \beta+1-2 / p}^{0}\left(\mathbb{R}^{3}\right)$ and $\theta: V_{2, \beta}^{0}\left(\mathbb{R}^{3}\right) \rightarrow V_{2, \beta}^{2}\left(\mathbb{R}^{3}\right)$ is the inverse to the operator of problem (4.16). Then (4.19) becomes the inequality (4.27). Then Lemma 4.9 implies 4.28), which is exactly 4.29. This concludes the proof.

LEMMA 4.11. Let the assumptions of Lemma 4.4 hold. Let $f \in V_{2, \varkappa}^{l}\left(\mathbb{R}^{3}\right) \cap$ $V_{p, \varkappa}^{l}\left(\mathbb{R}^{3}\right), p \geq 2$. Then for solutions $u \in V_{2, \varkappa}^{l+2}\left(\mathbb{R}^{3}\right)$ of problem 4.16 the following inequality is valid:

$$
\|u\|_{V_{p, \varkappa}^{l+2}\left(\mathbb{R}^{3}\right)} \leq c\|f\|_{V_{p, \varkappa}^{l}\left(\mathbb{R}^{3}\right)},
$$

where $c$ does not depend on $u$ and $f$.

Proof. From 4.7) with $\beta=\varkappa$ we have

$$
\|u\|_{V_{p, \varkappa}^{l+2}\left(\mathbb{R}^{3}\right)} \leq c\left(\|f\|_{V_{p, \varkappa}^{l}\left(\mathbb{R}^{3}\right)}+\|u\|_{V_{p, \varkappa-l-2}^{0}\left(\mathbb{R}^{3}\right)}\right) .
$$

From Lemma 4.10 and from the assumption that $f \in V_{p, \varkappa-l}^{0}$ we have $p(\beta+1)$ $-2=p(\varkappa-l)$, so $\beta=\varkappa-l-1+2 / p$, so $p(\beta-1)-2=p(\varkappa-l-2)$. Hence, 


$$
\|u\|_{V_{p, \varkappa-l-2}^{0}\left(\mathbb{R}^{3}\right)} \leq c\|f\|_{V_{p, \varkappa-l}^{0}\left(\mathbb{R}^{3}\right)} \leq c\|f\|_{V_{p, \varkappa}^{l}\left(\mathbb{R}^{3}\right)} .
$$

Thus 4.30 holds. This concludes the proof.

Theorem 4.1. Let $\operatorname{Im} \lambda=\beta-1 \notin \mathbb{Z}$. Let $f \in V_{p, \beta}^{l}\left(\mathbb{R}^{3}\right), l \in \mathbb{N}, p \in$ $(1, \infty), \beta \in \mathbb{R}$. Then there exists a solution to problem (4.1) such that $u \in$ $V_{p, \beta}^{l+2}\left(\mathbb{R}^{3}\right)$ and

$$
\|u\|_{V_{p, \beta}^{l+2}\left(\mathbb{R}^{3}\right)} \leq c\|f\|_{V_{p, \beta}^{l}\left(\mathbb{R}^{3}\right)},
$$

where $c$ does not depend on $u$ and $f$.

Proof. Let $\left\{f_{\nu}\right\}$ be a sequence of smooth functions with compact support in $\mathbb{R}^{3} \backslash L$ which converges to $f$ in $V_{p, \beta}^{l}\left(\mathbb{R}^{3}\right)$. By Lemma 4.5 there exists a solution $u_{\nu} \in V_{2, \beta}^{l+2}\left(\mathbb{R}^{3}\right)$ to problem 4.1 with r.h.s. $f_{\nu}$. From 4.30 the sequence $\left\{u_{\nu}\right\}$ converges in $V_{p, \beta}^{l+2}\left(\mathbb{R}^{3}\right)$. This concludes the proof.

\section{Existence in a bounded domain}

Proof of Theorem 1.1. To prove Theorem 1.1 we introduce a partition of unity (see [7]). We distinguish four types of subdomains: $\Omega^{(1)}$-near an interior point of $\Omega \cap L, \Omega^{(2)}$ —near the point where $L$ meets $S, \Omega^{(3)}$-near an interior point of $\Omega$ but at a positive distance from $L, \Omega^{(4)}$-near a point of $S$ at a positive distance from $L$. With each subdomain $\Omega^{(k)}, k=1,2,3,4$, we connect a smooth function $\zeta^{(k)}$ which is equal to 1 in $\bar{w}^{(k)} \subset \Omega^{(k)}$ and vanishes outside of $\Omega^{(k)}$.

We shall restrict our considerations to problem $(3.2)$ only, because problem 3.5 can be treated in the same way.

Let us extend the boundary condition $(3.2)_{2}$ by introducing a function $\tilde{b}$ such that

$$
\bar{n} \cdot \nabla \tilde{b}=b .
$$

Then the function

$$
u=\varphi-\tilde{b}
$$

is a solution to the problem

$$
\begin{aligned}
& \Delta u=f \quad \text { in } \Omega, \\
& \left.\bar{n} \cdot \nabla u\right|_{S}=0,
\end{aligned}
$$

where $f=-\Delta \tilde{b}$. Multiplying $(5.3)_{1}$ by $\zeta^{(1)}$ and defining $u^{(1)}=u \zeta^{(1)}, f^{(1)}=$ $f \cdot \zeta^{(1)}$ we obtain, instead of (5.3), the problem

$$
\begin{aligned}
& \Delta u^{(1)}=f^{(1)}+2 \nabla \zeta^{(1)} \nabla u+\Delta \zeta^{(1)} u, \\
& \left.u^{(1)}\right|_{\partial \Omega^{(1)}}=0 .
\end{aligned}
$$


Multiplying (5.3) by $\zeta^{(2)}$ we obtain

$$
\begin{array}{ll}
\Delta u^{(2)}=f^{(2)}+2 \nabla \zeta^{(2)} \nabla u+\Delta \zeta^{(2)} u & \text { in } \Omega^{(2)} \times(0, T), \\
\bar{n} \cdot \nabla u^{(2)}=u \bar{n} \cdot \nabla \zeta^{(2)} & \text { on } S \cap \bar{\Omega}^{(2)} .
\end{array}
$$

Let us introduce a local coordinate system $y=\left\{y_{1}, y_{2}, y_{3}\right\}$ with the origin at the point where $L$ meets $S$ and such that $y_{3}>0$ describes points inside $\Omega$. Let $S^{(2)}=S \cap \bar{\Omega}^{(2)}$ be described by the relation

$$
y_{3}=F\left(y_{1}, y_{2}\right) \text {. }
$$

Introducing new coordinates

$$
\begin{gathered}
z_{i}=y_{i}, \quad i=1,2, \\
z_{3}=y_{3}-F\left(y_{1}, y_{2}\right),
\end{gathered}
$$

we define a mapping $z=\Phi(y)$.

If problem 3.2 is formulated in coordinates $x=\left\{x_{1}, x_{2}, x_{3}\right\}$ we can pass to coordinates $y=\left\{y_{1}, y_{2}, y_{3}\right\}$ by a rotation and a translation. We denote this mapping by $y=Y(x)$. Hence

$$
\begin{aligned}
& z=(\Phi \circ Y)(x) \equiv \Psi(x), \\
& \hat{\Omega}^{(2)}=\Psi\left(\Omega^{(2)}\right), \quad \hat{S}^{(2)}=\Psi\left(S^{(2)}\right) .
\end{aligned}
$$

Introducing the notation

$$
\begin{aligned}
& \tilde{u}^{(2)}(z)=u^{(2)}\left(\Psi^{-1}(z)\right), \quad \tilde{u}(z)=u\left(\Psi^{-1}(z)\right), \\
& \nabla_{\Psi}=\left.\frac{\partial z}{\partial x}\right|_{x=\Psi^{-1}(z)} \cdot \nabla_{z}=\left.\Psi_{x}\right|_{x=\Psi^{-1}(z)} \cdot \nabla_{z}, \\
& \bar{n}_{z}=(0,0,1), \quad \bar{n}_{\Psi}=\left.\left(F_{y_{1}}, F_{y_{2}},-1\right)\right|_{y=\Phi^{-1}(z)},
\end{aligned}
$$

we can express problem 5.5 in the form

$$
\begin{array}{ll}
\nabla_{z}^{2} \tilde{u}^{(2)}=\left(\nabla_{z}^{2}-\nabla_{\Psi}^{2}\right) \tilde{u}^{(2)}+2 \nabla_{\Psi} \tilde{\zeta}^{(2)} \nabla_{\Psi} \tilde{u}+\nabla_{\Psi}^{2} \tilde{\zeta}^{(2)} \tilde{u} & \text { in } \hat{\Omega}^{(2)}, \\
\bar{n}_{z} \cdot \nabla_{z} \tilde{u}^{(2)}=\left(\bar{n}_{z}-\bar{n}_{\Psi}\right) \cdot \nabla_{z} \tilde{u}^{(2)}+\bar{n}_{\Psi} \cdot \nabla_{z} \tilde{\zeta}^{(2)} u & \text { on } \hat{S}^{(2)} .
\end{array}
$$

Let $\tilde{\eta}^{(2)}$ be a function such that

$$
\left.\frac{\partial}{\partial z_{3}} \tilde{\eta}^{(2)}\right|_{z_{3}=0}=\left(\bar{n}_{z}-\bar{n}_{\Psi}\right) \cdot \nabla_{z} \tilde{u}^{(2)}+\tilde{u} \bar{n}_{\Psi} \cdot \nabla_{z} \tilde{\zeta}^{(2)} .
$$

Then the function

$$
\tilde{\psi}^{(2)}=\tilde{u}^{(2)}-\tilde{\eta}^{(2)}
$$


is a solution to the problem

$$
\begin{aligned}
& \nabla_{z}^{2} \tilde{\psi}^{(2)}=\Delta \tilde{\eta}^{(2)}+\left(\nabla_{z}^{2}-\nabla_{\Psi}^{2}\right) \tilde{u}^{(2)}+2 \nabla_{\Psi} \tilde{\zeta}^{(2)} \nabla_{\Psi} \tilde{u} \\
& +\nabla_{\Psi}^{2} \tilde{\zeta}^{(2)} \tilde{u} \equiv \tilde{F}^{(2)} \quad \text { in } \hat{\Omega}^{(2)}, \\
& \left.\frac{\partial}{\partial z_{3}} \tilde{\psi}^{(2)}\right|_{z_{3}=0}=0 \text {, } \\
& \left.\tilde{\psi}^{(2)}\right|_{\partial \hat{\Omega}^{(2)} \backslash\left\{z: z_{3}=0\right\}}=0,
\end{aligned}
$$

where

$$
\hat{\Omega}^{(2)}=\left\{z \in \mathbb{R}^{3}:\left(z_{1}^{2}+z_{2}^{2}\right)^{1 / 2}<R, 0<z_{3}<a, \varphi_{z} \in[0,2 \pi]\right\},
$$

$\varphi_{z}=\operatorname{arctg}\left(\frac{z_{2}}{z_{1}}\right)$. After reflection with respect to the plane $z_{3}=0$ problem (5.12) assumes the form

$$
\begin{aligned}
& -\nabla_{z}^{2} \tilde{\psi}^{\prime(2)}=\tilde{F}^{\prime(2)}, \\
& \left.\tilde{\psi}^{\prime(2)}\right|_{\partial \hat{\Omega}^{\prime}(2)}=0,
\end{aligned}
$$

where $\eta^{\prime}$ means that $\eta^{\prime}\left(z^{\prime}, z_{3}\right)=\eta\left(z^{\prime}, z_{3}\right)$ for $z_{3}>0, z^{\prime}=\left(z_{1}, z_{2}\right)$ and $\eta^{\prime}\left(z^{\prime}, z_{3}\right)=\eta\left(z^{\prime},-z_{3}\right)$ for $z_{3}<0$, and

$$
\hat{\Omega}^{\prime}(2)=\left\{z \in \mathbb{R}^{3}:\left(z_{1}^{2}+z_{2}^{2}\right)^{1 / 2}<R,-a<z_{3}<a, \varphi_{z} \in[0,2 \pi]\right\} .
$$

Applying Lemma 4.11 we obtain for solutions to problems (5.4) and (5.13) the estimate

$$
\begin{aligned}
\left\|u^{(k)}\right\|_{V_{p,-\mu}^{l+2}(\Omega)} \leq & c\left\|f^{(k)}\right\|_{V_{p,-\mu}^{l}(\Omega)} \\
& +c\left(\|\nabla u\|_{V_{p,-\mu}^{l}\left(\Omega \cap \Omega^{(k)}\right)}+\|u\|_{V_{p,-\mu}^{l}\left(\Omega \cap \Omega^{(k)}\right)}\right),
\end{aligned}
$$

where $k=1,2$ and in the case of problem $(5.13)$ we used that $\operatorname{diam} \Omega^{(2)}$ is sufficiently small.

In the case of the subdomains $\Omega^{(3)}$ and $\Omega^{(4)}$ we obtain problems similar to (5.4) and (5.9). Then instead of (5.14) we get

$$
\left\|u^{(k)}\right\|_{W_{p}^{l+2}(\Omega)} \leq c\left\|f^{(k)}\right\|_{W_{p}^{l}(\Omega)}+c\left(\|\nabla u\|_{W_{p}^{l}\left(\Omega^{(k)}\right)}+\|u\|_{W_{p}^{l}\left(\Omega^{(k)}\right)}\right) .
$$

Moreover, for solutions to problem $(5.3)$ we have

$$
\|u\|_{W_{p}^{l+2}(\Omega)} \leq c\|f\|_{W_{p}^{l}(\Omega)} .
$$

Let $\mu \in(0,1)$. Then summing up inequalities (5.14) and (5.15) and applying the Hardy inequality with estimate (5.16) to the last two terms on the r.h.s. of (5.15) we obtain

$$
\|u\|_{V_{p,-\mu}^{l+2}(\Omega)} \leq c\|f\|_{V_{p,-\mu}^{l}(\Omega)} .
$$

Now let $\mu \in(1,2)$. Then the last two terms on the r.h.s. of $(5.14)$ can be estimated in view of the Hardy inequality and (5.17). Repeating the other arguments we obtain (5.17) for $\mu \in(1,2)$. 
Continuing the above considerations and assuming that $u \in V_{p,-\mu}^{l+2}(\Omega)$, $\mu \in(k-1, k)$ we obtain (5.17) for $\mu \in(k, k+1), k \in \mathbb{N}$.

Repeating the above considerations for problem (3.5) we obtain by (3.1) the following estimate for solutions to problem 1.1):

$$
\|v\|_{V_{p,-\mu}^{l+1}(\Omega)} \leq c\left(\|w\|_{V_{p,-\mu}^{l}(\Omega)}+\|b\|_{V_{p,-\mu}^{l+1-1 / p}(S)}\right) .
$$

This holds for smooth functions vanishing sufficiently fast near $L$.

To prove Theorem 1.1 we use Theorem 1.2 Let $\left(w_{\nu}, b_{\nu}\right)$ be a sequence of smooth functions with compact support in $\Omega \backslash L$ which converges to $(w, b) \in$ $V_{p,-\mu}^{k}(\Omega) \times V_{p,-\mu}^{k+1-1 / p}(S)$. In view of Theorem 1.2 we have the existence of an approximate solution of the problem

$$
\begin{aligned}
& \operatorname{rot} v_{\nu}=w_{\nu}, \\
& \operatorname{div} v_{\nu}=0, \\
& \bar{n} \cdot v_{\nu}=b_{\nu} .
\end{aligned}
$$

By Theorem 1.2 there exists a solution to problem (5.19) such that $v_{\nu} \in$ $H_{-\mu}^{l+2}(\Omega)$ with r.h.s. equal to $w_{\nu}$ and $b_{\nu}$. Using estimate (5.18) we find that the sequence converges in $V_{p,-\mu}^{l+2}(\Omega)$ and 1.4 holds. This concludes the proof.

Acknowledgments. This research is partially supported by MNiSW grant no 1 P03A 02130 and partially by EC FP6 Marie Curie ToK programme SPADE2.

\section{References}

[1] È. B. Bykhovskiı̆, Solvability of a mixed problem for the Maxwell equations for ideal conductive boundary, Vestnik Leningrad. Univ. 12 (1957), 50-66 (in Russian).

[2] N. Dunford and J. T. Schwartz, Linear Operators, Wiley-Interscience, 1966.

[3] O. A. Ladyzhenskaya, V. A. Solonnikov and N. N. Ural'tseva, Linear and Quasilinear Equations of Parabolic Type, Nauka, Moscow, 1967 (in Russian).

[4] V. G. Maz'ya and B. A. Plamenevskiı̌, $L_{p}$-estimates of solutions to elliptic boundary value problems in domains with edges, Trudy Moskov. Mat. Obshch. 37 (1978), 49-93 (in Russian).

[5] C. B. Morrey, Multiple Integrals in the Calculus of Variations, Springer, 1966.

[6] V. A. Solonnikov, Overdetermined elliptic boundary value problems, Zap. Nauchn. Sem. LOMI 21 (1971), 112-158 (in Russian).

[7] W. M. Zajączkowski, Existence of solutions to the (rot, div)-system in $L_{2}$-weighted spaces, Appl. Math. (Warsaw) 36 (2009), 83-106. 
Wojciech M. Zajaczkowski

Institute of Mathematics

Polish Academy of Sciences

Śniadeckich 8

00-956 Warszawa, Poland

E-mail:wz@impan.pl

and

Institute of Mathematics and Cryptology

Cybernetics Faculty

Military University of Technology

Kaliskiego 2

00-908 Warszawa, Poland

Received on 30.1.2008;

revised version on 4.11.2009 